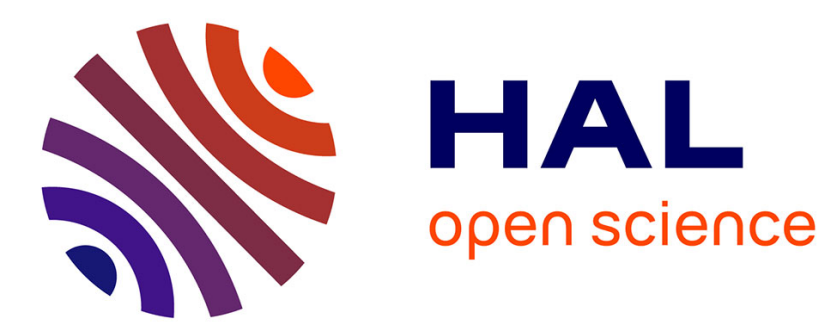

\title{
Le site Internet, centre de pilotage et d'investigation de la marque vers les micro-communautés virtuelles.
}

Franck Debos

\section{To cite this version:}

Franck Debos. Le site Internet, centre de pilotage et d'investigation de la marque vers les microcommunautés virtuelles.. Colloque international EUTIC2009 Enjeux et usages des TIC. Stratégies du changement dans les systèmes et les territoires, 2009, France. pp.1-18. sic_00439078

\section{HAL Id: sic_00439078 \\ https://archivesic.ccsd.cnrs.fr/sic_00439078}

Submitted on 5 Dec 2009

HAL is a multi-disciplinary open access archive for the deposit and dissemination of scientific research documents, whether they are published or not. The documents may come from teaching and research institutions in France or abroad, or from public or private research centers.
L'archive ouverte pluridisciplinaire HAL, est destinée au dépôt et à la diffusion de documents scientifiques de niveau recherche, publiés ou non, émanant des établissements d'enseignement et de recherche français ou étrangers, des laboratoires publics ou privés. 
Le site Internet, centre de pilotage et d'investigation de la marque vers les micro-communautés virtuelles.

\section{Franck DEBOS.}

Maître de Conférences Université de Nice Sophia-Antipolis.

debos.franck@wanadoo.fr

Laboratoire I3M, université de Nice Sophia-Antipolis.

UFR, Lettres, Arts et sciences Humaines.

98, Bd Édouard Herriot, B.P. 3209

06204 Nice Cedex.

\section{Résumé :}

Les marques doivent intégrer la volonté de participation des internautes au sein de leurs stratégies, volonté souvent relayée par de multiples blogs et communautés virtuelles. De ce fait, que le site Internet doit être la pierre angulaire de la stratégie de développement du territoire virtuel de la marque. Il peut être assimilé à un véritable " Hub Marketing » capable de piloter au mieux la destinée des marques.

Mots clés : Site Internet, Communautés Virtuelles, Marques, Consom'acteur, Stratégies Communicationnelles . 


\section{Introduction.}

Internet crée une transformation en profondeur des relations entre les individus et les marques par l'évolution du processus de transaction, de ses comportements et préférences.

Nous pouvons observer depuis la fin des années 1990 et l'émergence successive du consom'expert et du consom'acteur une véritable restructuration du rapport de forces entre les entreprises et le citoyen en faveur de ce dernier.

Les marques doivent tenir compte de la volonté de participation de l'individu au sein de leurs stratégies, volonté souvent relayée sur Internet par de multiples blogs et communautés virtuelles. Nommé « Convergence » aux EtatsUnis, ce mouvement met au centre de toute réflexion stratégique l'idée de participation entre des publics divers avec les entreprises et leurs marques (Maillet 2008).

Avec Internet la notion de CSP s'efface pour laisser la place à des logiques de groupes de pression et communautés virtuelles d'intérêt. C'est sur la toile via les blogs que vont s'exprimer sans contraintes les attentes et critiques des consommateurs vis à vis des marques.

Le consom'acteur devient donc plus que jamais essentiel dans la vie voire la survie des marques comme l'atteste les résultats du baromètre de l'écoute et de la participation des internautes Français mis en place par Thierry Maillet et Crmmetrix en mars 2007 (www.customerlistening.typepad.com ).

Le principal enseignement à tirer de cette étude est que les français connectés sont en forte attente de considération, de respect et d'écoute et qu'ils estiment que la grande majorité des marques affichent un déficit à ce niveau. Ils ont le sentiment d'une écoute intéressée voire d'une « fausse 
écoute " par ces dernières dont ils jugent la démarche marchande trop exacerbée.

L'objet de cette communication est de montrer que le site Internet doit être la pierre angulaire de la stratégie de développement du territoire virtuel de la marque. II peut être assimilé à un véritable « Hub Marketing » capable de piloter au mieux la destinée des marques.

Après avoir présenté dans une première partie l'évolution des relations « marques - consommateurs » sur le Web, nous nous attacherons à identifier des pistes de réflexions stratégiques au plan du marketing et de la communication permettant à une entreprise de renforcer le territoire virtuel de ses marques notamment par une cartographie très ciblée à partir des communautés virtuelles, de leur croisement ou de la mise en place d'une plate forme de blogs.

Pour ce faire, afin d'étayer nos propos, nous procèderons à une série d'entretiens semi-directifs auprès d'acteurs professionnels du secteur ; fournisseurs de solutions en Emarketing et E-Communication pour les entreprises. Les entretiens s'effectueront dans le cadre de deux salons professionnels sur ce thème, à savoir : le salon DEV COM et le premier forum Networking de la Côte d'Azur, tous deux se déroulant sur le site de Sophia-Antipolis. 


\section{I) Le consommateur « $\mathbf{2 . 0}$ » et les marques}

a) Du consommateur au consom'acteur.

Le fort développement d'Internet avec le Web 2.0 propose au consommateur une nouvelle façon d'appréhender le monde.

Au plan sociologique cela implique une évolution du prisme des réseaux sociaux dans lesquels s'insère l'individu avec le développement des communautés virtuelles s'ajoutant aux communautés humaines et remettant en question les concepts suivants :

- Le concept de lieu de vie lorsque l'on peut en de multiples endroits se connecter aux quatre coins du monde ou dans des univers virtuels (Jeux en lignes, Second life, Facebook) prenant une place aussi importante que le réel pour certains.

- La perception du temps quand l'on peut à tout moment appeler, écrire, acheter, discuter et se voir par écran interposé.

-La notion de groupe d'appartenance primaire ou secondaire par l'intégration à des dizaines de formes et de lieux de communautés virtuelles à géométrie variable et à responsabilités limitées. A ce niveau, nous entendons par la notion de groupes, des ensembles de personnes qui ont des caractéristiques similaires et qui obéissent à une dynamique spécifique dans leurs rapports sociaux. Ils possèdent leurs propres codes et exercent une pression de conformité auprès de leurs membres afin que soit respectées les croyances et les « lois » du groupe(J Brée 2004). - Les transmissions de savoir, de savoir-faire et la création artistique lorsque les élèves, les clients et les «visiteurs virtuels » peuvent modifier et détourner le contenu de cours ou d'œuvres d'art.

- Les notions de lieux d'achat avec le commerce et la visite de magasins et galeries marchandes virtuelles. 
- Le lieu du travail avec la décentralisation des décisions et des structures ainsi que le développement du télétravail.

Concomitamment, Internet entraîne aussi une évolution radicale des relations entre les consommateurs et les entreprises par la modification du processus de transaction, de ses comportements et de ses préférences. Au sein de cette nouvelle donne relationnelle, nous pouvons déterminer quatre facteurs rapprochant l'Internaute des entreprises et de leurs marques.

- La création de communautés marchandes et non marchandes ou autres réseaux sociaux liées aux caractéristiques propres $d^{\prime}$ Internet (connectivité, ubiquité, fluidité, rapidité et universalité).

- Une relative transparence de ce " cybermonde " renforcée par l'accès à une information plus large et plus disponible pour l'usager / consommateur sans toutefois négliger sur un plan opérationnel les difficultés de l'utilisateur peu expérimenté qui se retrouve dans les méandres du Web. Sur ce plan, le développement de « courtiers d'informations » ou de « métamédiaires » (F Jallat, M Haenlein 2006) peuvent apporter un début de réponse.

- La connaissance des motivations et freins de l'usager, du client ou du prospect se révèle un enjeu stratégique pour les entreprises (ou autres organisations développant des marques). En effet, dans un environnement virtuel, ces dernières interprètent et analysent le comportement de leur marché à venir auprès des individus. Dans un tel contexte, toute structure ne pouvant connaître et exploiter ces informations sera en proie à des difficultés importantes.

- L'individu veut devenir un véritable « consom'acteur » vis-à-vis de ses interlocuteurs humains ou institutionnels en s'intégrant toujours davantage dans la chaîne de valeurs de 
l'organisation. II désire s'impliquer directement au sein de la politique commerciale de l'entreprise et devient un partenaire à part entière pour cette dernière qu'il estime souvent obéissant à une logique marchande très forte et souhaite qu'elle adopte un comportement responsable. De plus on observe récemment que l'innovation partagée entre concepteur et utilisateur tend à de déplacer de plus en plus vers I'utilisateur final (P Musso, L Ponthou, E Seulliet 2007). L'illustration de l'intervention croissante des individus dans l'innovation technologique qui devient "ascendante " (J Tillinac 2006) est fournie par le Web 2.0 véritable espace public mondial de création et d'innovation sur Internet. En effet; une part non négligeable de l'innovation provient des consommateurs eux mêmes et non des laboratoires et centres de R\&D à l'instar des logiciels libres. Cette constatation coïncide avec le fait que la génération des "Digital Native » née dans les années 1970 et 1980 a grandi avec le phénomène numérique. Elle ne peut concevoir de vivre sans Internet, le téléphone portable ou le MP3. Dans ce contexte informationnel précis, le consommateur ou l'usager est également coproducteur puisque en utilisant cette information, il lui donne sa véritable valeur ajoutée.

Nous pouvons aux réflexions précédentes rajouter le fait que le développement d'Internet crée une interactivité électronique qui peut modifier le modèle de représentation du monde et pose la problématique du cadre d'interprétation de cette information. L'émetteur premier s'efface progressivement pour n'être identifié que comme le relais d'une information sans signataire, posant ainsi le problème de l'authentification de tout ce qui circule sur les réseaux.

Le développement d'Internet coïncide donc avec un besoin d'interactivité renaissant qui se manifeste fortement chez les 
individus et reflète un désir de considération, de participation à toutes les facettes de la vie sociale, culturelle, économique et politique de la société dans laquelle ils se situent.

Toutefois, le numérique initie de nouvelles contraintes dans les nouvelles relations individus - entreprises, à savoir :

- Une volatilité plus importante des clients ou des usagers qui peut être compensée par une personnalisation du service.

- Des craintes voire des peurs associées au problème de sécurisation des paiements ou au phénomène de «Phamming ». ${ }^{1}$ ou de « Phishing "

- L'immatérialité qui rend la notion de confiance incontournable dans les échanges électroniques.

- Le sentiment d'abandon devant le caractère parfois complexe et nébuleux du réseau. A cet état psychologique s'ajoutent les difficultés techniques et structurelles liées à Internet, d'où l'intérêt de créer des sites de plus en plus conviviaux rendant cet environnement virtuel accessible à tout usager quel que soit son profil.

En conclusion, nous pouvons dire que le numérique est un facteur clé de succès de la stratégie des entreprises et de ses marques à condition de savoir intégrer et impliquer un consommateur qui est à la fois cible et pilote.

\footnotetext{
${ }^{1}$ Il s'agit du détournement de la connexion de l'internaute afin de lui subtiliser son identifiant et mot de passe.

2 II s'agit de faux messages usurpant l'identité d'une entreprise (Banque, fournisseurs d'accès Internet, etc.) visant à essayer de récupérer des numéros de carte bancaires, de comptes, etc.
} 


\section{b) Marque Commerciale et Internet, une relation complexe}

Cette évolution du comportement du consommateur est à mettre en perspective avec le fait que la marque commerciale est devenue l'un des principaux vecteurs de l'image et de la responsabilité sociale de l'entreprise. Comme l'énonce J P Baudoin (2006) elle est « le signe de l'entreprise qui l'oblige à répondre aux exigences du citoyen autant que du consommateur $»$.

Par l'interface de la marque se crée une relation originale et spécifique entre les consommateurs et l'entreprise. Les Blogs (F Nonnenmacher 2006), forums de discussion, le Podcasting ou encore les flux RSS (real simple syndication) sont de nouveaux médias électroniques qui découlent de l'essor du numérique. Ils peuvent être considérés comme des canaux de communication innovants pour les marques sous conditions d'utilisation maîtrisée et pertinente. L'aspect novateur est ici plus lié à la manière dont se recherche l'information qu'aux qualités technologiques de ces outils de communication ce qui implique l'évolution d'une logique de marque normative ou « impérialiste » présentant, voire imposant un univers de vie au consommateur vers une logique de marque "plastique» qui va intégrer et respecter les codes du consommateur afin de mieux le séduire.

En mettant à disposition de multiples et combinés moyens d'expression des exigences individuelles; le numérique via Internet notamment renforce cette situation en permettant à l'individu qu'il soit client ou tierce personne de s'immiscer dans le fonctionnement de l'entreprise. De par sa fonction bijective, Internet brise le monopole des messages par les entreprises et les marques en offrant à chacun un espace de libre expression. Ayant à sa disposition des moyens conviviaux et ouverts, le 
consommateur peut modifier et détourner des textes, vidéos, images et sons traitant d'une marque spécifique.

La marque commerciale; principal actif immatériel de l'entreprise peut être de ce fait plus facilement discuté, critiqué et mis en cause, soit directement par le site Internet des entreprises et des marques, soit indirectement par le biais des sites ou des blogs de groupes de pression (association consuméristes, écologistes, ONG...).

II faut d'ailleurs savoir que comme l'énonce Sharon Greene (2008), directrice internationale de Risc International, une minorité d'internautes ${ }^{3}$ (environ 55 millions soit 4 pour cent de la population totale) influence 1,3 milliard de consommateurs et donc a un pouvoir de valorisation et de dénigrement certain sur les grandes marques commerciales. De façon générale, ces " accros créatifs » sont jeunes (moins de 30 ans) et impliqués au plan social et environnemental dans une optique très altruiste. Ils peuvent se rapprocher des "créatifs culturels » (J P Worms 2007) car ils sont non conformistes, se considèrent comme des leaders naturels et sont constamment à l'affût de nouveautés sur le net. Sur ce plan, ils sont pionniers dans les usages sur le Net et s'intègrent dans les processus d'innovation ascendante traité précédemment. En effet, ils participent au développement de nouveaux services qu'ils vont quitter dès que cesderniers sont utilisés par le plus grand nombre afin migrer de nouveaux univers qu'ils auront contribué à créer.

\footnotetext{
${ }^{3}$ II s'agit d'une enquête réalisé au dernier trimestre 2007 par le biais de questionnaires écrits (France et Chine) et en ligne (USA) auprès de :

- 3000 Chinois urbains de 15 à 65 ans avec une analyse centrée sur les moins de 30 ans (32\% de l'échantillon).

- 2500 Français urbains représentatifs de la population adulte avec une analyse centrée sur les moins de 30 ans ( $23 \%$ de l'échantillon).

- 3000 Américains représentatifs de la population avec une analyse centrée sur les moins de 30 ans (26\% de l'échantillon).
} 
Les marques ne peuvent les ignorer, d'autant plus qu'ils sont plutôt critiques vis à vis de ces dernières et peuvent réagir violemment à toute tentative de récupération ou de manipulation par le biais de faux blogs d'entreprises (appelés "flogs " ou "fake blog s " comme ce fut le cas de Sony en 2006). Toutefois, même si ils représentent un groupe de pression virulent, en tant que pionnier, ils ne reflètent presque jamais l'opinion de la majorité des internautes et des consommateurs dans leur ensemble. II faut donc, dans une logique de veille stratégique, les considérer comme des précurseurs, des "renifleurs de tendances" (C Michon, $\mathrm{O}$ Badot, J C Andréani 2006) pouvant préfigurer le comportement de la majorité des internautes de demain.

II) Le site Internet, point d'ancrage du territoire virtuel de la marque.

a) Méthodologie d'étude.

Notre recherche s'est scindée en deux étapes.

Dans un premier temps nous avons procédé à une douzaine d'entretiens semi directifs auprès des professionnels du secteur considéré dans le cadre de deux manifestations importantes sur la technopole de Sophia-Antipolis.

- Le salon DEVCOM Azur ${ }^{4}$ qui s'est déroulé le 24 mars 2009 (Six entretiens semi-directifs). II s'agit d'un évènement regroupant une cinquantaine d'exposants présentant une offre exhaustive des meilleures pratiques en E-technologie dans les secteurs de la communication, du marketing et de la vente. En outre, une trentaine de conférences et d'ateliers rencontres ont lieu sur l'ensemble des domaines concernés par ce salon.

\footnotetext{
${ }^{4}$ www.devcom-azurexpo.com
} 
- Le premier Forum Networking de la Côte d'Azur qui a eu lieu le Jeudi 14 mai 2009 au CERAM à Sophia-Antipolis ${ }^{5}$ (Six entretiens semi-directifs) sur le thème des réseaux socioprofessionnels au service de l'entreprise. L'objectif était de faire une évaluation de l'importance des réseaux sociaux et professionnels en termes d'optimisation des relations intra et interprofessionnelles par une approche multidisciplinaire constructive au sein de l'entreprise. L'adoption d'une stratégie d'intégration et de développement de ces réseaux permet de renforcer la relation client, la performance commerciale et la productivité de l'entreprise.

Ces entretiens ont été complétés par la participation à des groupes de discussion lors de la première édition du BarCamp Sophia-Antipolis le samedi 20 Juin à l'Agora Einstein sur le thème de la gestion de l'identité numérique et l'e-réputation et plus spécifiquement sur la notoriété d'un individu ou d'une entité dans le monde virtuel. II s'agit d'une journée rencontre entre professionnels et amateurs de TIC toutes catégories socioprofessionnelles confondues dans le cadre d'ateliers interactifs ou tous participent dans un contexte convivial et informel.

\section{b) Quel territoire virtuel pour les marques ?}

D’après les résultats des entretiens menés, il apparaît que les critères de segmentation classiques comme les CSP par exemple sont de moins en moins fiables au profit d'une logique de classification par micro-communautés d'intérêts et groupe de pression. Les internautes sont de véritables consom'acteurs dans la mesure ou ils vont choisir d'être des clients en répondant ou non aux sollicitations des marques sur le Web. Ce nouveau comportement implique une évolution dans le positionnement et les stratégies commerciales et

\footnotetext{
${ }^{5}$ www.forum-networking-cote-azur.com
} 
communicationnelles des marques ce qu'apparemment une majorité de responsables marketing (notamment au niveau des PME) n'ont pas complètement intégré. II est essentiel de communiquer directement avec les communautés virtuelles de consommateurs, de les informer sur la réelle valeur ajoutée de la marque considérée, d'avoir un discours sérieux, transparent et de proximité afin de faire des internautes de véritables ambassadeurs de cette marque sur la toile. Cependant, plusieurs entreprises ont encore une stratégie de marque peu interactive, voire "impérialiste" (Chauveau 2002) et ne valoriseront pas leurs atouts au détriment des concurrents, notamment des marques de distributeurs dont les enseignes ont compris l'enjeu stratégique d'être bien positionnées sur le Web. De plus, indépendamment d'un coût très avantageux (Une année de présence et d'interactivité permanente sur Internet est en moyenne identique au prix de deux passages publicitaires en prime time sur TF1 - Cerf 2008), le Web permet d'associer du contenu avec l'audio et la vidéo et de mesurer précisément les contacts et la traçabilité. Internet va donc renforcer l'imaginaire et le territoire de la marque.

A ce niveau, il peut être intéressant de prendre en compte les multiples communautés d'internautes réunis autour de marques comme Nutella, Nestlé, Louis Vuitton, etc. et de les croiser afin de créer un effet de levier et d'identifier des profils de consommateurs qu'il faudra séduire et fidéliser. En tant qu'espace de libre expression, le Web diffuse les réelles attentes et critiques autour d'une marque, notamment via certains blogs. De ce fait il peut être pertinent de mettre en place au sein de l'entreprise une structure de type observatoire effectuant une analyse des contenus des blogs qui traitent de sa ou ses marques. Dans une optique similaire, il semble pertinent d'élaborer une plate-forme de blogs autour d'une marque afin de bâtir une cartographie d'internautes très 
ciblée et de sélectionner des "leaders d'opinion »de cette marque sur la toile afin d'engendrer un buzz positif.

Dans la mesure où les consommateurs sont « naturellement » attirés par les sites Internet des marques, ceux ci deviennent des points centraux déterminants de la stratégie et des plans marketing de l'entreprise. Ce constat est corroboré par les répondants de l'étude qui précisent que les internautes les plus en empathie avec la marque vont être de véritables leaders d'opinion communicants pour cette dernière. Ils désireront pour la plupart d'entre eux donner leurs impressions sur les produits de l'entreprise et les tester. L'entreprise doit de ce fait développer une stratégie d'écoute à deux niveaux :

- Une stratégie d'écoute "active » ou "dynamique» qui implique la mise en place d'un véritable dialogue avec le consommateur et la réponse aux éventuelles critiques.

- La mise en place d'une démarche "netnographique » dans laquelle l'entreprise observer et apprendre des internautes en s'immergeant dans leur conversations. II s'agit d'une logique d'écoute "passive " dans laquelle marques et entreprises vont écouter et suivre les buzz (notamment des blogs les plus virulents) afin d'adapter leur démarche communicationnelle et commerciale vis à vis des consommateurs. II faut avoir présent à l'esprit qu' Internet, ceci étant accentué par le Web 2.0, est un réseau de conversation mondial et puissant et les marques qui seront le mieux positionnés sur leur marché dans un futur proche seront celles qui auront les meilleures facultés d'écoute, d'intégration, d'apprentissage et d'interaction à partir des discours tenus sur le Web par les blogueurs ou simple internautes ${ }^{6}$.

\footnotetext{
${ }^{6}$ Sur ce plan, il peut être opportun de lire « The Cluetrain Manifesto » de Christopher Locke, Rick Levine, Doc Searles et David Weinberger (Janvier 2000, 224 pages). Les auteurs montrent qu'avec le Web dès la fin des
} 
Toutefois, même s'il peut être tentant de vouloir intégrer des blogueurs ou autres contributeurs actifs dans le processus d'innovation et de test des produits d'un marque à cause de leur impact certain sur l'opinion des internautes, il faut bien avoir présent à l'esprit que ces derniers ne sont pas représentatifs de l'ensemble du marché mais plutôt des comportements émergents au sein de celui ci. II faut de ce fait mettre en perspective par rapport à une appréciation globale leurs avis et discours d'autant plus qu'ils sont soupçonneux par définition et attentifs à tout risque de manipulation ou de tromperie.

Par le fait que leur capacité de fragiliser la marque est aussi forte que leur apport sur ce plan, l'entreprise doit être très prudente quand à sa logique de partenariat vis à vis de ce type d'internaute. II faut également relativiser cette situation dans la mesure où ces groupes d'influenceurs se renouvellent constamment sur la toile.

Une autre réflexion provenant des personnes interrogées qui est importante pour la stratégie de l'entreprise est que même si Internet propose à un annonceur une grande variété de moyens pour amplifier sa notoriété et valoriser son offre commerciale (Bandeaux contextuels, bannières publicitaires sur des sites spécialisés, sites communautaires et d'enchères ou encore blogs thématiques, e-mailing personnalisées, etc.), celui ne doit pas être le seul support. En effet, le consommateur est aujourd'hui «multi écran » par le biais de la télévision, du téléphone et d'Internet et amplifie la fragmentation de l'audience avec une attention sans cesse volatile.

années 1990, les individus créent de nouvelles façons de partager les informations qui les intéressent de manière exponentielle. De ce fait les marchés en réseau se structurent plus vite, sont mieux informés et plus « intelligents » que la plupart des entreprises qui les fournissent et qui sont présentes sur la toile. 
L'entreprise doit donc élaborer une stratégie de communication qui reste plurimédias avec des supports très complémentaires pour pérenniser la réputation de sa ou ses marques, le tout complété par une analyse poussée de la connaissance des clients afin de bien déterminer le cœur de cible ou les "pionniers", d'optimiser le plan de communication média et hors média, en réduisant la déperdition des messages à la portion congrue et en lui permettant surtout d'avoir une offre adaptée à ses tendances.

\section{Conclusion: Le site Internet, tête de pont de la stratégie communicationnelle de la marque.}

Comme nous l'avons vu précédemment, c'est maintenant le consommateur qui vient à la marque lorsqu'il désire une relation privilégiée et spécifique. Le volet qualitatif de ce contact va donc primer par rapport à des critères quantitatifs. Dans ce contexte, les sites de marques deviennent des points d'ancrage à forte valeur ajoutée qui vont attirer les meilleurs clients de la marque et/ou les leaders d'opinion? ${ }^{7}$.

La stratégie communicationnelle de l'entreprise doit avoir dans ce cas un contenu riche afin de personnaliser et pérenniser ses relations avec cette catégorie de clients pour obtenir un contact de qualité. Cette orientation stratégique se justifie également par une concurrence accrue des marques de distributeurs qui pousse les " grandes » marques à développer une relation plus intime avec leurs clients.

A l'inverse de la rigidité des médias classiques en termes d'exposition, le site Internet de la marque est un point de convergence «naturel» et indispensable pour les

\footnotetext{
${ }^{7}$ D'après la banque de données SiteCRM de Crmmetrix regroupant plus de 6 millions de cas observés, $85 \%$ des visiteurs d'un site correspondent à des prospects ou clients impliqués et ayant des affinités importantes avec la marque considérée et/ou le segment de marché dans lequel elle est positionnée.
} 
consommateurs qu'il s'agisse de simplement s'informer sur la marque, échanger des informations ou développer une relation d'échange plus approfondie.

A la lumière des entretiens effectués et de nos lectures, nous pouvons considérer que le site de marque est le point de contact qui permet le meilleur retour sur investissement en tant que relais d'ensemble de sa stratégie de communication. Par son intermédiaire, le consommateur va être incité à devenir un véritable consom'acteur qui pourra créer un " buzz » positif autour de la marque voire même s'investir plus en co-créant et inventant avec la marque en fonction de son profil. Ces consom'acteurs seront concepteurs et/ou diffuseur. En effet, le site de la marque attire des personnes aux profils complémentaires qui peuvent être de véritables créatifs (Rogers 1995, Gladwell 2006) comme des leaders d'opinion que l'on peut identifier. Ceux-ci peuvent être intégrés à chaque étape du développement du produit et de la stratégie de communication externe de l'entreprise qu'elle soit institutionnelle ou commerciale.

Quelque soit le cas de figure, le site de marque joue un rôle déterminant dans l'attraction et le recrutement de ces personnes et la diffusion d'un contenu viral. Grace à son site l'entreprise, sa ou ses marques pourront communiquer avec le consommateur plutôt que seulement vers ce dernier.

\section{BIBLIOGRAPHIE :}

BAUDOIN Jean Pierre., "Marques \& TIC, quelle vitalité ! ", La Revue des Marques, 2006, $n^{\circ}$ 54, p 6-8.

BREE Joël., Le comportement $d u$ consommateur, Paris, Editions Dunod, 2004,128 pages. 
CERF Olivier, "Le sur mesure, point de passage ", La Revue des Marques, 2008, $n^{\circ} 64$, p $80-82$.

CHAUVEAU Alain "La Marque Impérialiste est Morte", La Revue des Marques, 2002, $n^{\circ} 7$, p 40-42.

FLORES Laurent « 10 facts about the value of brand websites », Paris, AdMap, World Advertising Research Center, 2004, p 26-28.

GLADWELL Malcolm, Le point de bascule, Montréal, Editions Transcontinental, 2006, 255 pages.

GREENE Sharon, "Dynamique d'influence sur le Web », RISC White Paper, 2008, 9 pages.

GREENFIELD Adam, " Every[ware], la révolution de l' Ubimédia » Limoges ,Editions FYP, Collection Innovation, 2007, 256 pages.

JALLAT Frédéric, HAENLEIN Michaël, Développer un marketing en ligne, dans MICHON C, Le Marketeur, Paris, Editions Pearsons Education, 2006, p 347-369.

JAWORSKI Bernard, KHOLI Ajay, ARVIND Sahay, « Market -Driven versus driving markets», Journal of the Academy of Marketing Science,2000, 28, 1, p 45-54.

LOCKE Christopher, LEVINE Rick, SEARLES Doc, WEINBERGER David, The Cluetrain Manifesto, Cambridge, Massachusset, Edition Perseus Book, 2000, 224 pages.

MAILLET Thierry, "Génération participation: de la société de consommation à la société de la participation", Paris, Editions MM2, 2008, 249 pages.

MICHON Christian, BADOT Olivier, ANDREANI Jean-Claude, «Comprendre les marchés: Connaissance Qualitative et quantitative ", p 31-57, dans MICHON C, Le Marketeur, Paris, Editions Pearsons, 2006, p 31-57. 
MUSSO Pierre, PONTHOU Laurent, SEULLIET Eric, Fabriquer le futur 2, Paris, Coll. Le Village Mondial, Editions Pearsons Education, 2007, 307 pages.

NONNENMACHER François, Blogueur d'entreprise, Paris, Editions Organisation, 2006, 268 pages

PEPPERS Don, ROGERS Martha, Le one to one : valorisez votre capital client, Paris, Editions d’Organisation, 1999, 312 pages.

ROGERS Everett, Diffusion of innovations, New York, The Free Press, 1995, 518 pages

TILLINAC Jean, "Le Web 2.0 ou l'avènement du client ouvrier ", Revue Quaderni, Paris, Editions Sapientia 2006, $n^{\circ} 60$.

WORMS Jean-Pierre Les créatifs culturels en France, Association pour la biodiversité culturelle (Préface de JP Worms), 2007, Coll. Société Civile Edition Yves Michel, 132 pages. 